\title{
Hormone-refractory Prostate Cancer
}

Treatment options for patients with hormone-refractory disease remain limited and include palliation of symptoms (especially pain) and/or systemic cytotoxic chemotherapy. However, because of up to now limited efficacy, the use of cytotoxic chemotherapy has not been routine practice. Single agent chemotherapy has been associated with relevant palliative effects but no single agent has been associated with an objective response rate greater than $30 \%$. Palliative effects have been observed in HRPC patients following the administration of corticosteroids, mitoxantrone with either prednisone or hydrocortisone.

Based on results from two phase III randomized trials, the combination of mitoxantrone with corticosteroids was recognized as the reference treatment in metastatic HRPC [1]. Until recently, these hormonerefractory patients have been thought to be resistant to cytotoxic agents and, prior to 2004 , no randomized trial of chemotherapy in patients with androgen-independent prostate cancer (AIPC) had demonstrated a survival benefit.

\section{New Chemotherapy era in prostate cancer}

Docetaxel in phase I and II trials exhibited a significant activity in various dosing regimen in the range of 40 to $75 \mathrm{mg} / \mathrm{m}^{2}$ for the conventional three-weekly schedule and in the range of 20 to $40 \mathrm{mg} / \mathrm{m}^{2}$ for the weekly schedule. Evidence of this activity included PSA decline, objective response in bidimensionally measurable lesions and pain control. The safety profile was assessed as acceptable throughout these studies with a good risk/benefit ratio in this clinical setting. Now, however, the small yet significant survival benefit seen with docetaxel-based combinations has set a new standard in the treatment of patients with AIPC [2,3]. Current efforts are now being directed at identifying novel agents with activity in taxaneresistant populations, and at the development of agents with less toxicity than the current combination regimens. Although the combination of docetaxel and estramustine showed a survival benefit compared with the prior standard of mitoxantrone and prednisone,[2] researchers are now investigating less toxic biologic agents, such as calcitriol, as a replacement for estramustine; when combined with cytotoxic agents, these biologic agents increase the activity of the chemotherapy by inhibiting prosurvival signaling pathways [4]. Similarly, novel cytotoxic agents such as the epothilones are also being explored; this class of agents has demonstrated activity in taxane-resistant preclinical models [5]. Both of these strategies have shown promising results over the past year.

\section{Novel targeted therapies in HRPC}

Recent advances in our understanding of the pathophysiology of the disease have led to the identification of a number of rational prostate-specific drug targets. Novel agents that inhibit many of these targets have now been developed. These include the tyrosine kinase inhibitors (TKI), the endothelin receptor antagonist atrasentan; the therapeutic vaccines as Provenge (APC8015) and GVAX; monoclonal 
antibodies to prostate-specific membrane antigen (PSMA), and other ongoing new strategies as the antisense bcl-2 or the new monoclonal Pertuzumab (that inhibits her-2 heterodimerization).

\section{Inhibitors of kinase signaling pathways (TKI) in HRPC}

Because prostate cancer cells, particularly those present in bone, express high levels of activated PDGFR, imatinib was tested for activity in patients with AIPC. In a phase 1 study conducted by Mathew and colleagues, 28 patients with AIPC were treated with imatinib alone for 30 days at a dose of $600 \mathrm{mg}$ followed by imatinib $600 \mathrm{mg}$ in combination with docetaxel [6]. The lead-in design allowed for an assessment of imatinib single-agent activity in these patients, and demonstrated that two $(7 \%)$ of 28 patients had PSA declines $>50 \%$ on imatinib alone. Significantly greater activity was observed with the combination of imatinib and docetaxel, with eight (38\%) of 21 patients showing PSA declines $>50 \%$ and six additional patients $(29 \%)$ showing PSA declines $<50 \%$. In some patients, the responses were durable, lasting for greater than 18 months [6]. These data have led to a randomized phase 2 trial sponsored by the SPORE clinical consortium evaluating docetaxel and imatinib vs docetaxel and placebo in patients with AIPC. In contrast to the promising activity reported with imatinib and docetaxel, a second study testing the combination of imatinib $400 \mathrm{mg} /$ day and zoledronic acid in patients with AIPC was stopped early because of a lack of activity [7]. Overall, the experience with this agent is that it has minimal activity as a single agent in patients with prostate cancer but warrants further testing in combination with docetaxel [8].

The EGFR tyrosine kinase is expressed in $40 \%$ to $80 \%$ of prostate cancer cells, and increased expression of this target correlates with higher Gleason score and progression from an androgen-dependent to an androgen-independent state [9]. Results from one trial, a randomized, double-blind, placebo-controlled phase 2 trial of 58 patients, found that gefitinib (a TKI of EGF receptor (Her-1)) had only modest activity in delaying PSA rise[10]. The second trial, a randomized phase 2 study of 40 patients, found that it had little activity in patients with AIPC: no objective or PSA responses were observed [11]. Nevertheless, despite the limited activity of these agents as mono- therapy, combination trials of gefitinib and erlotinib with docetaxel and mitoxantrone are underway.

\section{Proteasome Inhibitors}

Proteasome inhibition thwart activation of the transcription machinery involved in proteins critical to tumor growth and metastases [12]. Bortezomib, a boronic acid dipeptide, is the first proteasome inhibitor to be investigated in humans $[12,13]$. This agent was approved in 2003 for use in patients with refractory multiple myeloma. A phase 1 dose-escalation trial of bortezomib in patients with solid tumors, including prostate cancer, showed that once-weekly bolus intravenous administration of bortezomib for 4 of 5 weeks is generally well tolerated; a dose of $1.6 \mathrm{mg} / \mathrm{m} 2$ was recommended for phase 2 testing [13]. Of the 48 patients in the trial with AIPC, some antitumor activity was evident in a minority of patients (eg, stable or declining PSA and reduction in lymphadenopathy). In a phase $1 / 2$ trial recently reported, Dreicer and colleagues randomized 32 patients to the combination of bortezomib $1.3 \mathrm{mg} / \mathrm{m} 2$ administered on days 2 and 9 plus docetaxel $40 \mathrm{mg} / \mathrm{m} 2$ administered on days 1 and 8 of a 21-day cycle [14]. Of the 25 patients evaluable for response, six (24\%) showed a PSA decline $>50 \%$, none of whom had undergone prior taxane therapy, while 3 of $13(23 \%)$ with measurable disease showed a partial response[14]. Further study with this combination and with other combinations will help determine the role of bortezomib in patients with AIPC.

\section{Endothelin-1 Inhibitors}

Endothelin-1 (ET-1) is a potent vasoconstrictor that modulates cell growth and proliferation, inhibits apoptosis, and stimulates osteoblast activity upon binding with the ET-A receptor $[15,16]$. In patients with prostate cancer, ET-1 and the ET-A receptor are overexpressed, and ET-1 degradation is downregulated, both of which occur in direct relation to advancing tumor stage, grade, and metastases [15-17]. Data establish ET-1 inhibition via ET-A blockade as an attractive target for therapy in patients with metastatic prostate cancer to bone [16]. Atrasentan is a highly potent selective ET-A receptor antagonist and represents the first ET-A receptor inhibitor to be tested clinically in prostate cancer [18-20]. Atrasentan has completed randomized, placebo-controlled clinical phase 2 and phase 3 studies in men with asymptomatic AIPC with time to progression as the clinical end point. The 
phase 2 randomized, controlled trial evaluated the activity of $2.5 \mathrm{mg}$ or $10 \mathrm{mg}$ of atrasentan in patients with metastatic AIPC. In this study of 288 patients, a significantly prolonged median time to disease progression (196 days vs 129 days, respectively; $P=.021)$ and a significantly longer median time to PSA progression (155 days vs 71 days, respectively; $\mathrm{P}=.002$ ) were observed in evaluable patients enrolled on the 10-mg atrasentan arm $(n=84)$ vs the placebo arm $(n=104)$ [21]. Results from the recently reported phase 3 trial evaluating the 10-mg dose of atrasentan $(n=408)$ vs placebo $(n=401)$ in patients with metastatic AIPC continued to show beneficial results in favor of atrasentan, although the primary end point of disease progression (ie, new lesions, clinical symptoms, skeletal complications, or pain) did not reach statistical significance in the intent-to-treat analysis [22]. Nevertheless, increases in bone alkaline phosphatase, total alkaline phosphatase, and PSA were significantly reduced in patients treated with atrasentan, suggesting that this agent delays disease progression [22]. As with the earlier trial, the most common adverse events were rhinitis, headache, and peripheral edema.

Results of a meta-analysis of atrasentan trials in 1097 patients showed a significant increase in the time to disease progression with atrasentan $(P=.013)$, which translated into a $19 \%$ reduction (hazard ratio 1.19) in the risk of disease progression. Significant decreases were also seen in the incidence of and the onset to pain in the atrasentan vs placebo groups $(P=.003)$.

The results from these trials demonstrate a clear benefit for the use of atrasentan in the management of metastatic AIPC. On the basis of these results, the manufacturer of atrasentan, has announced its intention to submit the agent for FDA approval. A phase 3 trial of $10 \mathrm{mg}$ atrasentan in men with nonmetastatic AIPC is still ongoing but closed to patient accrual.

\section{Angiogenesis Inhibitors}

\section{Bevacizumab}

In patients with metastatic prostate cancer, plasma VEGF levels are significantly higher than in patients with localized disease. This increase in VEGF levels is particularly pronounced in patients with PSA levels of greater than $20 \mathrm{ng} / \mathrm{m}$ [23]. Using archival serum samples from patients enrolled on CALGB study 9480 , a phase 3 Intergroup study of suramin, it has been demonstrated that plasma VEGF levels inversely correlate with survival time in patients with metastatic AIPC. In a multivariate model, VEGF levels remained significant factors for survival at various cut points tested [24]. Similarly, studies of the same dataset showed that pretreatment urine levels of VEGF were predictive of survival [25]. These data suggest that angiogenic inhibitors may be effective agents in prostate cancer; trials of bevacizumab and other novel angiogenesis inhibitors are ongoing. $A$ study by CALGB investigated the addition of bevacizumab to standard docetaxel and estramustine chemotherapy in patients with progressive metastatic AIPC (CALGB 90006) [26]. Seventy-nine patients were treated with estramustine $280 \mathrm{mg}$ TID days 1-5; docetaxel $70 \mathrm{mg} / \mathrm{m} 2$ on day 2; and bevacizumab $15 \mathrm{mg} / \mathrm{kg}$ over 30 minutes on day 2 . Standard dexamethasone premedication for docetaxel was given, and warfarin $2 \mathrm{mg}$ daily was administered with the goal of reducing thromboembolic complications associated with the use of estramustine. Posttherapy PSA declines were seen in 58 of $72(81 \%)$ patients, with a median time to objective disease progression of 9.7 months and an overall median survival of 21 months, results that compare favorably with other docetaxel-based combinations [26]. A randomized, double-blinded, placebo-controlled phase 3 trial comparing docetaxel and prednisone with and without bevacizumab in patients with AIPC is scheduled to begin enrollment this year.

\section{Thalidomide}

The underlying mechanism of thalidomide remains unknown, but the agent has anti-nflammatory, immunomodulatory, and antiangiogenic properties that likely account for its antitumor effects [27] Inhibition of VEGF, basic fibroblast growth factor (bFGF), interleukin (IL)-6, and tumor necrosis factor (TNF)alpha is believed to underlie its antiangiogenic effects $[27,28]$. Thalidomide has been tested in patients with prostate cancer and has shown encouraging early results. A randomized, dose-escalation phase 2 trial of thalidomide monotherapy was performed in 63 patients with AIPC. Fifty men received thalidomide at a dose of $200 \mathrm{mg} /$ day and an additional 13 men received escalating doses, ranging from 200 to 1200 $\mathrm{mg} /$ day. Declines in PSA of $>/=50 \%$ were observed 
in $18 \%$ of patients in the low-dose arm, while no responses were observed in the high-dose arm $[27,29]$. In a second phase 2 study investigating the use of thalidomide at a dose of $100 \mathrm{mg} /$ day for up to 6 months in men with AIPC, declines in PSA $>/=50 \%$ were observed in only three of the $20(15 \%)$ patients. Although the response rate was low, the responses were sustained for the treatment duration [28]. Common side effects, reported from the phase 2 studies, included constipation, drowsiness, fatigue, rash, and peripheral sensory neuropathy $[28,29]$. As with bevacizumab, thalidomide is also being studied in combination with cytotoxic chemotherapy. A randomized phase 2 study compared docetaxel monotherapy with docetaxel plus thalidomide at a dose of $200 \mathrm{mg} /$ day in patients with chemotherapy-naive metastatic AIPC [30]. In this trial, $53 \%$ of patients treated with the combination showed a PSA decline $>/=50 \%$ vs $37 \%$ in the docetaxel-only arm. Median progression-free survival was 5.9 months in the combination arm vs 3.7 months in the docetaxel-only arm $(P=$ not significant) $[30,31]$. These data suggest that although the activity of thalidomide as a single-agent therapy in prostate cancer may be limited, its use in combination with standard chemotherapy is promising.

\section{Immunotherapy in HRPC}

\section{Vaccines and Antibodies}

Several therapeutic vaccines for prostate cancer have demonstrated encouraging preliminary results and are now in advanced stages of clinical testing [32]. Provenge (an autologous CD54-positive dendritic cell vaccine loaded with a recombinant fusion protein consisting of prostatic acid phosphatase and granulocyte macrophage-colony stimulating factor (GM-CSF) and GVAX, are currently in phase 3 clinical testing. Numerous other approaches are being evaluated in earlier stage clinical trials in men with hormone-naive and hormone-refractory disease .

Dendritic cells are immune cells that present antigens to other components of the immune systems. Favorable phase 2 trial results in patients with AIPC[33] led to a randomized, placebo-controlled phase 3 clinical trial in the same patient population. Preliminary findings from the phase 3 trial, conducted in 127 asymptomatic men with metastatic disease, revealed a trend toward a delay in disease progression among study participants immunized with the vac- cine [34]. In an unplanned subgroup analysis, it was found that vaccination with Provenge significantly delayed time to disease progression and time to onset of disease-related pain in patients with intermediate and lower grade disease (Gleason score $</=7)$. The vaccine is also being tested in combination with bevacizumab in a phase 2 trial of patients with hormone-sensitive prostate cancer. A second vaccine, GVAX, which uses allogeneic cultured prostate cancer cells that have been modified to secrete high levels of GM-CSF, was shown to be immunogenic and generally well tolerated in phase 2 studies $[35,36]$. No dose-limiting toxicities were observed in a 24-week dose-escalation trial that included a prime-boost strategy in patients with metastatic AIPC [36]. One of the phase 2 trials showed that immunization with the vaccine is associated with a median survival of 26 months,[35] and the other, larger trial showed stable or decreasing levels of a biomarker of osteoclast activity in the majority of patients [36]. GVAX will be evaluated in two phase 3 trials in symptomatic and asymptomatic patients with metastatic AIPC and any Gleason score. Antibodies currently being investigated for prostate cancer include those that target tumor-associated antigens, such as mucin and PSMA $[37,38]$, cell surface receptors such as EGFR, and immunostimulatory molecules such as CTLA-4 [39], an antigen expressed on T cells.

\section{Other ongoing strategies}

Other strategies that are in development include: Antisense molecules against bcl-2 (Genasense) with or without chemotherapy [40], monoclonal antibodies against her-2 (Herceptin) and others preventing heterodimer formation of Her-2 (pertuzumab or 2C4 McAb) [41,42], anti Cox-2 agents, anti PI3K-Akt, the use of osteoprotegerin, the use of McAb against the RANKL (avoiding the ligand of RANKL to its receptor) and several others.

\section{Conclusions}

The increasing number of new therapies that are emerging for the treatment of prostate cancer, particularly for AIPC, is encouraging. New insights into cancer biology and immunology in general and in prostate cancer tumor biology and pathogenesis in particular, have accelerated research efforts aimed at bringing new prostate cancer therapies to physicians 
and patients. The large and growing number of potential therapies currently in clinical testing will hopefully translate into an increase in the number of effective treatment options available for prostate cancer patients with advanced disease. Given the morbidity and mortality associated with advanced prostate cancer, and the very high incidence and increasing prevalence of this disease, continued research focused on discovering new targets and new therapies for all types of patients in all stages of disease is clearly needed.

\section{References}

1. Tannock IF, Osoba D, Stocker MR et al. Chemotherapy with mitoxantrone plus prednisone or prednisone alone for symptomatic hormone-resistant prostate cancer: a Canadian randomized trial with palliative end points. J Clin Oncol 1996; 14:1756-1764.

2. Petrylak DP, Tangen C, Hussain M, et al. SWOG-99-16: randomized phase III trial of docetaxel (D)/estramustine (E) versus mitoxantrone $(M) /$ prednisone $(p)$ in men with androgen-independent prostate cancer (AIPCA). Proc Am Soc Clin Oncol. 2004; 23:2. Abstract 3.

3. Eisenberger MA, De Wit R, Berry W, et al. A multicenter phase III comparison of docetaxel $(D)+$ prednisone $(P)$ and mitoxantrone (MTZ) + $\mathrm{P}$ in patients with hormonerefractory prostate cancer (HRPC). Proc Am Soc Clin Oncol. 2004; 23: 2. Abstract 4.

4. Beer TM, Eilers KM, Garzotto M, et al. Weekly high-dose calcitriol and docetaxel in metastatic androgen-independent prostate cancer. J Clin Oncol. 2003; 21: 123$-128$.

5. Sepp-Lorenzino L, Balog A, Su DS, et al. The microtubule-stabilizing agents epothilones $A$ and $B$ and their desoxy-derivatives induce mitotic arrest and apoptosis in human prostate cancer cells. Prostate Cancer Prostatic Dis. 1999; 2: 41-52.

6. Mathew P, Thall PF, Jones D, et al. Platelet-derived growth factor receptor inhibitor imatinib mesylate and docetaxel: a modular phase I trial in androgen-independent prostate cancer. J Clin Oncol. 2004; 22: 3323-3329.

7. Tiffany NM, Wersinger EM, Garzotto M, Beer TM. Imatinib mesylate and zoledronic acid in androgen-independent prostate cancer. Urology. 2004; 63: 934-939.

8. Rao KV, Goodin S, Capanna T, et al. A phase II trial of imatinib mesylate in patients with PSA progression after local therapy for prostate cancer. Proc Am Soc Clin Oncol. 2003; 22: 409. Abstract 1645.

9. Syed $\mathrm{S}$, Tolcher A. Innovative therapeutics for prostate cancer treatment. Rev Urol. 2003; 5(suppl 3): S78-S84.

10. Schroder FH, Wildhagen MF. ZD1839 (gefitinib) and hormone resistant (HR) prostate cancer - final results of a double blind randomized placebo-controlled phase II study. Program and abstracts of the 40th Annual Meeting of the American Society of Clinical Oncology; June 5-8, 2004; New Orleans, Louisiana. Abstract 4698.

11. Moore $M$, Winquist $E$, Pollak $M$, et al. A randomised phase II study of two doses of ZD1839 in patients (pts) with hormone refractory prostate cancer (HRPC): A NCl Canada Clinical Trials Group Study. Ann Oncol. 2002; 13(suppl 5): 90. Abstract 3260.

12. Mitchell BS. The proteasome - an emerging therapeutic target in cancer. N Engl J Med. 2003; 348: 2597-2598.

13. Papandreou CN, Daliani DD, Nix D, et al. Phase I trial of the proteasome inhibitor bortezomib in patients with advanced solid tumors with observations in androgen-independent prostate cancer. J Clin Oncol. 2004; 22 : 2109-2121.

14. Dreicer R, Roth B, Petrylak D. Phase $\mathrm{I} / \mathrm{II}$ trial of bortezomib plus docetaxel in patients with advanced androgen-independent prostate cancer. Proc Am Soc Clin Oncol. 2004; 23: 418. Abstract 4654.

15. Nelson J, Bagnato A, Battistini B, et al. The endothelin axis: emerging role in cancer. Nat Rev Cancer. 2003; 3: 110-116.

16. Carducci MA, Nelson JB, Bowling K, et al. Atrasentan, an endothelin-receptor antagonist for refractory adenocarcinomas: safety and pharmacokinetics. J Clin Oncol. 2002; 20: 2171-2180.

17. Gohji K, Kitazawa S, Tamada H, Katsuoka Y, Nakajima M. Expression of endothelin receptor a associated with prostate cancer progression. J Urol. 2001; 165: 10331036.

18. Nelson JB, Hedican SP, George DJ, et al. Identification of endothelin-1 in the pathophysiology of metastatic adenocarcinoma of the prostate. Nat Med. 1995; 1: 944-949 .

19. Nelson JB, Chan-Tack K, Hedican SP, et al. Endothelin-1 production and decreased endothelin $\mathrm{B}$ receptor expression in advanced prostate cancer. Cancer Res. 1996; 56: 663-668.

20. Yin JJ, Mohammad KS, Kakonen SM, et al. A causal role for endothelin-1 in the pathogenesis of osteoblastic bone metastases. Proc Natl Acad Sci U S A. 2003; 100 : 10954-10959.

21. Carducci MA, Padley RJ, Breul J, et al. Effect of endothelin-A receptor blockade with atrasentan on tumor progression in men with hormone-refractory prostate cancer: a randomized, phase II, placebocontrolled trial. J Clin Oncol. 2003; 21: 679-689.

22. Carducci M, Nelson JB, Saad F, et al. Effects of atrasentan on disease progression and biological markers in men with metastatic hormone-refractory prostate cancer: phase 3 study. Program and abstracts of the 40th Annual Meeting of the American Society of Clinical Oncology; June 5-8, 2004; New Orleans, Louisiana. Abstract 4508. 
23. Duque JL, Loughlin KR, Adam RM, Kantoff PW, Zurakowski D, Freeman MR. Plasma levels of vascular endothelial growth factor are increased in patients with metastatic prostate cancer. Urology. 1999; 54: 523-527.

24. George DJ, Halabi S, Shepard TF, et al. Prognostic significance of plasma vascular endothelial growth factor levels in patients with hormone-refractory prostate cancer treated on Cancer and Leukemia Group B 9480. Clin Cancer Res. 2001; 7: 1932-1936.

25. Bok RA, Halabi S, Fei DT, et al. Vascular endothelial growth factor and basic fibroblast growth factor urine levels as predictors of outcome in hormone-refractory prostate cancer patients: a cancer and leukemia group B study. Cancer Res. 2001; 61: 2533-2536.

26. Picus J, Halabi S, Rini B, et al. The use of bevacizumab $(B)$ with docetaxel $(D)$ and estramustine $(E)$ in hormone refractory prostate cancer (HRPC): initial results of CALGB 90006. Proc Am Soc Clin Oncol. 2003; 22: 393. Abstract 1578.

27. Franks ME, Macpherson GR, Figg WD. Thalidomide. Lancet 2004; 363: 1802-1811.

28. Drake $\mathrm{MH}$, Robson W, Mehta P, et al. An open-label phase II study of low-dose thalidomide in androgenindependent prostate cancer. $\mathrm{Br} \mathrm{J}$ Cancer. 2003; 88: 822-827.

29. Figg WD, Dahut W, Duray P, et al. A randomized phase II trial of thalidomide, an angiogenesis inhibitor, in androgen-independent prostate cancer. Clin Cancer Res. 2001; $7:$ 1888-1893.

30. Dahut WL, Gulley JL, Arlen PM, et al. Randomized phase II trial of docetaxel plus thalidomide in androgen-independent prostate cancer. J Clin Oncol. 2004; 22: 25322539.

31. Figg WD, Arlen P, Gulley J, et al. A randomized phase II trial of docetaxel (taxotere) plus thalidomide in androgen-independent prostate cancer. Semin Oncol. 2001; 28: 62-66.

32. Fong L, Small EJ. Immunotherapy for prostate cancer. Semin Oncol. 2003; 30: 649-658.

33. Burch PA, Croghan GA, Gastineau DA, et al. Immunotherapy (APC8015, Provenge(R)) targeting prostatic acid phosphatase can induce durable remission of metastatic androgen-independent prostate cancer: a phase 2 trial. Prostate. 2004; 60: 197-204.

34. Kylstra JW, Nemunaitis J, Small EJ, et al. A placebo-controlled phase 3 trial of immunotherapy (APC8015, Provenge(R)) for androgen independent prostate cancer (AIPC): Evidence that Gleason score predicts immunologic as well as clinical responses to therapy. Program and abstracts of the American Association for Cancer Research 95th Annual Meeting; March 27-31, 2004; Orlando, Florida. Abstract 1408.

35. Simons J, Nelson W, Nemunaitis J, et al. Phase II trials of a GM-CSF gene-transduced prostate cancer cell line vaccine (GVAX) in hormone refractory prostate cancer. Proc Am Soc Clin Oncol. 2002; 21: 183a. Abstract 729.

36. Small E, Higano C, Smith D, et al. A phase 2 study of an allogeneic GM-CSF gene-transduced prostate cancer cell line vaccine in patients with metastatic hormone-refractory prostate cancer (HRPC). Proc Am Soc Clin Oncol. 2004; 23: 396. Abstract 4565.

37. Bander NH, Nanus DM, Milowsky MI, et al. Targeted systemic therapy of prostate cancer with a monoclonal antibody to prostate-specific membrane antigen. Semin Oncol. 2003; 30: 667-677.

38. Milowsky MI, Nanus DM, Kostakoglu L, et al. Phase I trial of yttrium-90-labeled anti-prostate specific membrane antigen monoclonal antibody J591 for androgen-independent prostate cancer. J Clin Oncol. 2004; 22: 1-10.

39. Fong L, Rini B, Kavanaugh B, et al. CTLA-4 blockade-based immunotherapy for prostate cancer. Proc Am Soc Clin Oncol. 2004; 23: 185. Abstract 2590.

40. Gleave M, Tolcher A, Miyake, et al: Progression to androgen independence is delayed by adyuvant treatment with antisense bcl-2 oligodeoxynucleotides after castration in the LNCaP prostate tumor model. Clin Cancer Res 1999; 5: 2981-2988.

41. Agus DB Scher HI, Higgins B et al; Response of prostate cancer to anti-Her-2/neu antibody in androgen-dependent and independent human xenograft models. Cancer Res 1999; 59: 4761-4,

42. Agus DB, Akita RW, Fox WD, et al. Targeting ligand-activated ErbB2 signaling inhibits breast and prostate tumor growth. Cancer Cell. 2002; 2: 127-37. 